\title{
Doped and Multilayer Biocompatible Materials Prepared by Hybrid Laser Deposition
}

\author{
Miroslav Jelinek $^{1,2}$, Tomas Kocourek ${ }^{1,2}$, Jan Remsa ${ }^{1,2}$, Petr Pisarik ${ }^{1,2}$, Jan Miksovsky ${ }^{1,2}$ \\ ${ }^{1}$ Institute of Physics of the Czech Academy of Sciences, Na Slovance 2, 18221 Prague 8, Czech Republic. \\ ${ }^{2}$ Czech Technical University in Prague, Faculty of Biomedical Engineering, nam. Sitna 3105, 27201 Kladno, \\ Czech Republic.
}

* Corresponding author. Tel.: +420 26605 2733; e mail : jelinek@fzu.cz

Manuscript submitted January 10, 2018; accepted April 18, 2018.

doi: 10.17706/ijbbb.2018.8.4.252-258

\begin{abstract}
Hybrid laser technology for synthesizing doped, nanocomposite, single and multilayers of amorphous or nanocrystalline materials are presented. The technique use method of pulsed laser deposition (PLD) or combination of PLD with magnetron sputtering, radiofrequency discharges or two PLD running together. Examples of deposition and study of doped biocompatible materials as Cr: DLC, Ti: DLC, Ag: DLC, Ag: hydroxyapatite, and enhancement of sp3- DLC layers bonds by in situ ion gun bombardment are given.
\end{abstract}

Key words: PLD, hybrid PLD, biocompatible coating, implants.

\section{Introduction}

Biomaterials, i.e. natural or artificial materials designed for application in biotechnologies and medicine, are widely used for replacing irreversibly damaged tissues in the human body. Biomaterials have to meet the requirements for well-functioning and long-term durability of the implants, namely biocompatibility, good corrosion and fatigue resistance, wear resistance and biomechanical compatibility [1]. In many cases a single coating material alone cannot fulfil the technical requirements. To improve physical, mechanical and biocompatible properties of implant coatings, the layers can be doped (enriched) with other elements.

The cheapest way for laser fabrication of doped biocompatible coating is to cover the rotating target material (matrix) with a piece of dopant material. But in present study of layers, multilayers and superlattices of doped biocompatible materials the focus is on increasing flexibility and range of deposition conditions by way of combining PLD with magnetron sputtering (MS), PLD with RF discharges, ion gun layers modification and on the combination of two simultaneously running PLD systems (dual PLD). The methods allow carefully study the influence of dopation on a large scale of dopants and to create nanocomposite biomaterials of new properties [2]

Experiences with deposition of chromium, silver and titanium doped diamond-like carbon (DLC) and hydroxyapatite (HA) films and ferroelectric BaTiO3 multilayers for coating of metallic bone implants are presented. Layers of different dopants concentration and ferroelectric- based multilayers were prepared. Overwiev of our research in that direction and the results of physical and biomedical analysis are presented in summary.

\section{Experimental}


The doped and multilayer biocompatible coatings were prepared by PLD, PLD combined with radiofrequency discharges (RF), PLD with magnetron sputtering (MS), PLD with ion bombardment, and by dual PLD. The two KrF excimer lasers (LUMONICS PM 842, $\lambda=248 \mathrm{~nm}, \tau=20 \mathrm{~ns}$ and COMPexProTM $205 \mathrm{~F}$, $\lambda=248 \mathrm{~nm}, \tau=20 \mathrm{~ns}$ ) were used. (Fig. 1) The energy density of laser beam on the target ranged from 2 to $16 \mathrm{Jcm}^{-2}$, repetition rate of pulses were from 1 to $50 \mathrm{~Hz}$, argon atmosphere pressure from $10^{-4} \mathrm{~Pa}$ (vacuum) to $1 \mathrm{~Pa}$. Thin layers of DLC were deposited on fused silica (FS), Si (100) or metallic substrates. The substrate deposition temperature for DLC films was $\mathrm{T}_{\mathrm{S}}=20^{\circ} \mathrm{C}$. The target-substrate distance $\left(\mathrm{D}_{\mathrm{T}-\mathrm{s}}\right)$ was $45 \mathrm{~mm}$ for PLD, $85 \mathrm{~mm}$ for MS, and $260 \mathrm{~mm}$ for ion bombardment. DC magnetron (K.J.Lesker TRS2CF) was set for deposition process with power from 0 to $200 \mathrm{~W}$. For in situ bombardmen of DLC grown films the ion gun eH200 (Kaufman and Robinson, Inc.) was used. The operating parameters of the ion gun were held on working pressure of $0.25 \mathrm{~Pa}$ or $0.5 \mathrm{~Pa}$, cathode current of $0.15 \mathrm{~A}$ or $0.5 \mathrm{~A}$ and energy of ions was changed from $30 \mathrm{eV}$ to $210 \mathrm{eV}$. Before the deposition process the substrates were cleaned by RF discharge (13.56 $\mathrm{MHz}$ ) in $5 \mathrm{~Pa}$ of argon for two minutes [3]-[5].

Morphology - Thickness and roughness were measured by an Alpha-step IQ mechanical profilometer (KLA TENCOR Co.), and the layer roughness was examined by AFM.

The crystalline properties were measured by XRD (X-ray diffraction). Parallel beam geometry, detector scan with the stationary sample and the glazing angle of incidence (GAOI) was used.

The concentration of elements was determined using WDX measurement (WDX - wavelength dependence X-ray analysis) was analysed with EDAX Jeol Supersprobe 733. The composition of layers and the content of trace elements were determined by Particle Induced X-ray Emission, and Rutherford Backscattering Spectrometry methods using 3 MV Tandetron 4130MC accelerator at INP Rez with the energy of protons equal to $2.94 \mathrm{MeV}$.

X-ray photoelectron spectra (XPS) were measured by an ADES-400 photoelectron spectrometer (VG Scientific, UK) using Mg Ka radiation (1253.6 eV). The spectra were recorded for wide-survey and narrow scans in C $1 \mathrm{~s}$ and $01 \mathrm{~s}$ regions with a pass energy of $100 \mathrm{eV}$ or $20 \mathrm{eV}$ (C $1 \mathrm{~s}$ line). Inelastic electron background was subtracted using Shirley's procedure.

Adhesion - For determination of adhesion were used macroscratch tester REVETEST (CSM Instruments co.). The REVETEST system is a standard tester which is compatible with DIN EN 1071-3 and ASTM C1624. We used acoustic emission to estimate the delamination point and we visually confirmed it from the photography of the scratch. Rockwell diamond with tip radius of $200 \mu \mathrm{m}$ was used as indenter.
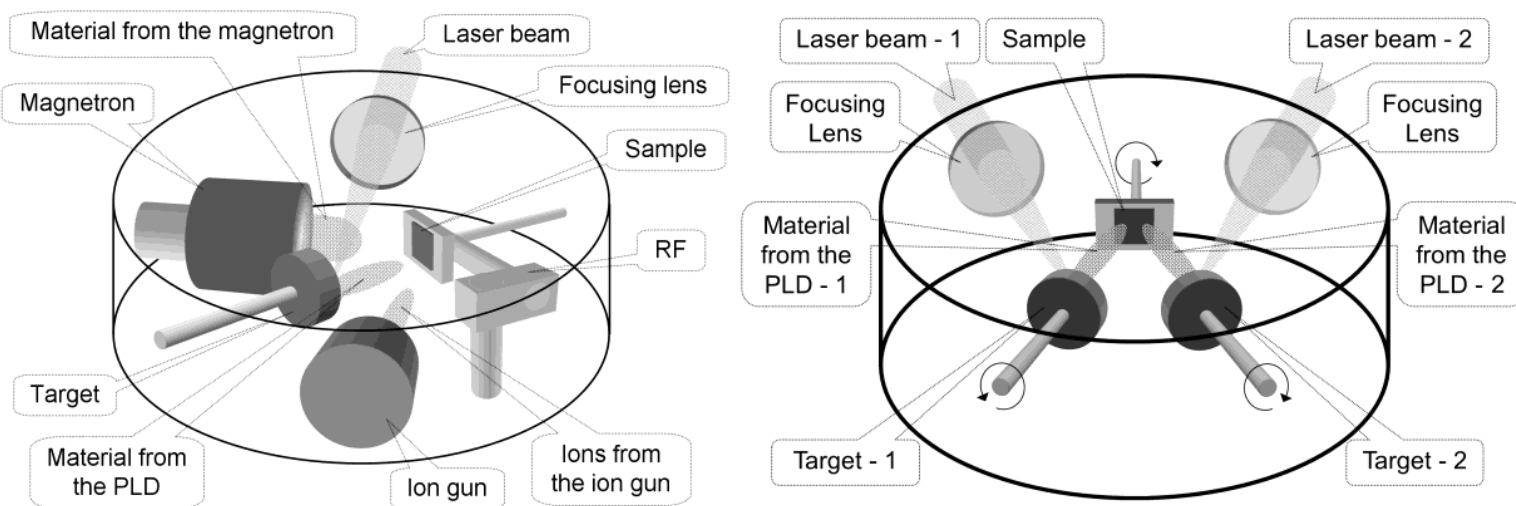

Fig. 1 a) Scheme of the PLD deposition system with radiofrequency discharges, magnetron sputtering and ion bombardment. b) Scheme of the dual PLD deposition system.

\section{Results and Discussion}




\subsection{Ag: HA Layers (For Improvement of Antibacterial Properties)}

The silver doped HA layers with dopation of 0.06 at $\%, 0.3$ at $\%, 1.2$ at $\%, 4.4$ at $\%, 8.3$ at $\%$ and 13.7 at $\%$ were fabricated by PLD method. Depend on deposition conditions the films were amorphous or polycrystalline. We observed a distinct difference in Young modulus $\mathrm{Y}$ and hardness $\mathrm{H}$ for amorphous and polycrystalline HA and Ag + HA films and the decrease in Y and H with silver dopation. No influence of crystallinity on bacterial efficacy was observed. Amorphous and polycrystalline hydroxyapatite and doped polycrystalline layers were non- toxic. Silver doped amorphous HA layers were mildly toxic from $1.2 \mathrm{at} . \% \mathrm{Ag}$. [6].

\subsection{Ag: DLC Films (For Improvement of Antibacterial Properties)}

Silver doped diamond-like carbon layers were deposited by dual pulsed laser deposition using two $\mathrm{KrF}$ excimer lasers. The concentration of Ag, determined by XPS and WDS, moved from zero to $\sim 10$ at. \%. Sp ${ }^{3}$ content in the Ag-doped DLC films decreased significantly with increasing Ag content in the films. AFM and SEM showed the pure DLC to be very smooth but an increasing roughness with rising silver content. The hardness (Storage modulus) changed from $51.9 \mathrm{GPa}$ to $26.0 \mathrm{GPa}$ (from $270.6 \mathrm{GPa}$ to $180.2 \mathrm{GPa}$ ) as a percentage of $\mathrm{Ag}$ content increased. The adhesion of layers to the titanium substrates improved with increasing Ag doping from $15 \mathrm{~N}$ to $21 \mathrm{~N}$. The contact angle measurements for water showed that the contact angle of Ag-DLC films $\left(78^{\circ}-98^{\circ}\right)$ was higher than that of the DLC film $\left(77^{\circ}\right)$. Antibacterial efficiency Ag-doped DLC films was evaluated with Pseudomonas aeruginosa and Staphylococcus aureus which frequently cause medical device-associated infections, e.g. bone implants. The experimental results showed that antibacterial efficiency increased to $99 \%$ with increasing the silver content in the films. [7]

\subsection{Cr: DLC Coating (For Better Adhesion)}

Chromium is used as a dopant for improvement of adhesion of DLC films. Chromium is also a common part of stainless steels and alloys used for implants (up to $30 \mathrm{wt} \%$ ) [4]. Chromium-doped diamond-like carbon (DLC) layers were prepared by a hybrid technology using a combination of PLD and magnetron sputtering ( 2 inch. K. J. Lesker) or PLD and PLD (double PLD deposition-two KrF excimer lasers were used).

\subsubsection{Cr: DLC prepared by double PLD deposition}

One $\mathrm{KrF}$ excimer laser was used for ablation of carbon and the second for ablation of chromium. The flows of targets materials were regulated by lasers repetition rates. Chromium content was in region from zero to 18 at\%. Roughness was studied by mechanical profilometer or by AFM. Roughness of doped films was comparable with the roughness of undoped DLC films. With increase of chromium content the roughness was slightly increasing [8] or decreasing [9]. Roughness Ra increased from about $0.62 \mathrm{~nm}$ to 1 $\mathrm{nm}$ (or decreased from $10 \mathrm{~nm}$ to $5 \mathrm{~nm}$ ]. With increasing chromium content the hardness changed from 52 GPa to $17 \mathrm{GPa}$ but adhesion critical force Lc3 increased from $6 \mathrm{~N}$ to $19 \mathrm{~N}$ [8], [9]. The contact angle (CA) of undoped DLC was $70.6^{\circ}$. For chromium doped layers changed from $87^{\circ}$ to $93^{\circ}$. Surface free energy (SFE) gamma T for undoped DLC layers was $43 \mathrm{mN} / \mathrm{m}$ and decreased for chromium doped layers to $31 \mathrm{mN} / \mathrm{m}$. No significant changes of SFE were observed for doped layers, the same is valid for zeta potential. Bacterial efficiency was low for all doped (undoped) layers [9].

\subsubsection{Cr: DLC prepared by PLD + magnetron sputtering}

Content of chromium was changed by magnetron power and of carbon by laser repetition rate. Measured chromium content in DLC layers was different for XPS measurement (in region from zero to 7 at\%) and Wavelength dependence X-ray analysis measurement (zero to 16 at\%). Films were very smooth. No reasonable differences in morphology were found between undoped and doped layers. Roughness Ra was in region from $0.2 \mathrm{~nm}$ to $0.7 \mathrm{~nm}$ (highest chromium concentration). Contact angle for water changed from 
$79^{\circ}$ (DLC) to $102^{\circ}$ (7 at $\%$ of $\mathrm{Cr}$ ). The SFE decreased with chromium doping from $34 \mathrm{~J} \cdot \mathrm{m}^{-2}$ (DLC) to $28 \mathrm{~J} \cdot \mathrm{m}^{-2}$ (7 at $\% \mathrm{Cr}$ ). The films do not exhibit any antimicrobial effect against both tested (positive and negative) bacterial strains [10]. From X-ray photoelectron spectroscopy follows that the chromium was present predominantly in its trivalent form. This form has been considered as relatively non toxic. Small amount of toxic, hexavalent, chromium was also found. The population density of Saos-2 cells was the highest in samples containing higher concentrations of chromium 7.7 and 10 at\%. However, this seemingly positive cell behavior could be associated with the risk of dedifferentiation and oncogenic transformation of cells [11], [12].

\subsection{Ti: DLC Coating}

Another element, which can be applied to lower the internal stresses and improve adhesion of the DLC to the substrate is titanium [13]. Titanium is also considered as good biocompatible material itself and titanium oxide support cells adhesion [14] and in higher concentration works as antibacterial agents [15].

We prepared thin films by two hybrid laser technologies: Dual pulsed laser deposition (Dual PLD) and PLD in combination with magnetron sputtering (PLD/MS). We focused to concentration of titanium in layers from zero up to 25 at.\% and compared results from both of the methods. Contents of the titanium dopant in the DLC layers were determined by WDX and confirmed by XPS. DualPLD technique seems to be better to reach the desired dopants concentration than PLD/MS.

The XRD tests shows that all the samples were amorphous. For the higher Ti content (10 and 25 at.\%) small amount of Ti and TiC phases were detect. Both methods produced smooth layers around $1 \mathrm{~nm}$, just for dualPLD method roughness increased for 10 and 25 at.\% Ti up to 5 resp. $15 \mathrm{~nm}$. Nevertheless some droplets up to hundreds of $\mathrm{nm}$ in high are presented on the surface, with more frequency for dualPLD and higher Ti concentration. Wearability tests have been done for loads $1 \mathrm{~N}$ and $2 \mathrm{~N}$. For these loads, there were no damage observed on samples. The wearability of the ball decreased with increased amount of titanium in layers. From comparison of the coated and uncoated materials, the uncoated materials were seriously damaged. The friction for pure DLC layers was $0.125 \pm 0.05$. For dual PLD method we observed slight increase in friction for lower concentration up to 0.14 and then decrease to 0.09 . The contact angle for water was in range from $57.7^{\circ}$ to $71.5^{\circ}$ for dual PLD method and from $53.8^{\circ}$ to $70.0^{\circ}$ for PLD/MS method. CA for ethylene glycol was in range from $34.5^{\circ}$ to $42.8^{\circ}$ resp. from $30.1^{\circ}$ to $44.0^{\circ}$ and for diiodomethane the range was from $34.9^{\circ}$ to $38.3^{\circ}$ resp. $32.0^{\circ}$ to $36.4^{\circ}$ for dual PLD resp. PLD/MS method. The results showed no trends in CA in correlation with titanium content in layers. From the free surface energy calculation we can conclude that all the tested layers are dominantly disperse. The hardness for pure DLC layers starts at (31 \pm 1 ) GPa and was decreasing with increasing of the titanium content to $12 \pm 2 \mathrm{GPa}$ for 25 at.\% of titanium. The decrease was slightly quicker for PLD/MS technique. The same behaviour we observed for elastic modulus with the highest values around $250 \mathrm{GPa}$ for pure DLC and decrease to $150 \mathrm{GPa}$ for 25 at.\% Ti, practically same for both methods. The adhesion values seemed to increase with content of the titanium with exception for highest concentration of titanium for PLD/MS method. Critical force $\mathrm{F}_{\mathrm{C} 3}$ started at $5.6 \mathrm{~N}$ for pure DLC and increased to $18.4 \mathrm{~N}$ for dualPLD technique for 25 at.\% Ti. For PLD/MS technique critical force was increasing up to $20.2 \mathrm{~N}$ for 10 at.\% Ti but for 25 at.\% Ti it reduced to $9.2 \mathrm{~N}$. This could be connected with lower hardness of titanium.

\subsection{Ion Bombarded DLC and Ti: DLC}

Bombarded DLC layers were prepared by PLD and Ti: DLC layers by PLD combined with magnetron sputtering. Both types of layers were bombarded during film growth. Influence of ions of argon [16], [17], xenon, nitrogen and oxygen were tested [17], [18] with focus on changes od sp $\mathrm{s}^{2}$ and $\mathrm{sp}^{3}$ bonds , film adhesion, hardness and biological properties. Argon bombarded layers exhibited changes of $\mathrm{sp}^{3}$ bonds [16]. 
For Ti: DLC layers the increase in $\mathrm{sp}^{3}$ bonds content reached up to $83 \%$.

\section{6. $\mathrm{BaTiO}_{3} / \mathrm{Pt} / \mathrm{TiNb}$ Multilayers}

The piezoelectric behaviour of collagen and the movement of ionic fluids are known to make the bones electrically active under mechanical loading. Correspondingly, electrically active materials are a desirable addition to implants. Moreover, such materials may improve healing and adaptation of the surrounding tissue. We studied ferroelectricity and biomedical properties of $\mathrm{BaTiO}_{3} / \mathrm{Pt}$ and $\mathrm{Pt} / \mathrm{BaTiO}_{3} / \mathrm{Pt}$ multilayers prepared by laser on fused silica substrates or TiNb implants. Crystalline BTO was prepared for large scale of deposition conditions (substrate temperature from $600^{\circ} \mathrm{C}$ to $750^{\circ} \mathrm{C}$, oxygen pressure in region $10 \mathrm{~Pa}$ to 20 Pa). For study of bio- properties the FS was used as a substrate (non-toxic, cheap) instead of TiNb implants. Pt buffer layer exhibited a positive impact on the growth of crystalline BTO. Films were smooth. Ferroelectricity was confirmed by Raman scattering and by electrical measurement methods on all crystalline films [19], [20]. For study of bio-properties the ferroelectric and non-ferroelectric BTO/Pt/FS layers were prepared. Influence of Pt on adhesion, crystallinity and bio- properties was tested on Pt/FS multilayers. Ferroelectric BTO layer supported the growth and osteogenic differentiation of human osteoblast-like Saos-2 cells in comparison with non-ferroelectric BTO layer.

\section{Conclusions}

Hybrid laser technologies show suitable and fast tool for study of new types of biocompatible coatings. By combination of PLD with magnetron sputtering and ion gun the influence of various dopands on the improvement of coating properties can be studied on large scale with a fine tuning of dopands concentrations. Samples and multilayers can be simply prepared with relatively cheap technology and small consumption of target materials. In the contribution the examples of technology and some of reached results are demonstrated on the synthesis and study of films like Ag doped hydroxyapatite and Ag, chromium and titanium doped DLC. Influence of ion bombardment and application of ferroelectric multilayers on implants behaviour is alos given.

\section{Acknowledgement}

The work was supported by the Grant Agency of Czech Technical University in Prague, grant No. SGS16/190/0HK4/2T/17 and by the Grant Agency of the Czech Republic (grant GA-15-01558S and grant GA15-05864S).

\section{References}

[1] Geetha, M., Singh, A. K., Asokamani, R., \& Gogia, A. K. (2009). Ti based biomaterials, the ultimate choice for orthopaedic implants - A review. Progress in Materials Science, 54(3), 397-425.

[2] Jelínek, M., Zemek, J., Remsa, J., Mikšovský, J., Kocourek, T., Písařík P., Trávníčková M., Filová, E., \& Bačáková, L. (2017). Hybrid laser technology and doped biomaterials. Applied Surface Science, 417(30), 73-83.

[3] Jelinek, M., Kocourek, T., Kadlec, J., \& Zemek, J. (2009). Hybrid laser-magnetron technology for carbon composite coating. LASER PHYSICS, 19(2), 149-153.

[4] Jelinek, M., Weiserova, M., Kocourek, T., Zezulova, M., \& Strnad, J. (2011). Biomedical properties of laser prepared silver-doped hydroxyapatite. LASER PHYSICS, 21(7), 1265-1269.

[5] Kocourek, T., Jelinek, M., Kadlec, J., Popov, C., \& Santoni, A. (2007). Thin TiCN films prepared by hybrid magnetron-laser deposition. Plasma Processes and Polymers, 4(1), S651-S654.

[6] Jelinek, M., Kocourek, T., Remsa, J., Weiserová, M., Jurek, K., Mikšovský, J., Strnad, J., Galandáková, A., \& Ulrichová, J. (2013). Antibacterial, cytotoxicity and physical properties of laser - Silver doped 
hydroxyapatite layers. Materials Science and Engineering: C, 33(3), 1242-1246.

[7] Písařík, P., Jelínek, M., Remsa, J., Mikšovský, J., Zemek, J., Jurek, K., Kubinová, Š., \& Šepitka, J. (2017). Antibacterial, mechanical and surface properties of Ag-DLC films prepared by dual PLD for medical applications. Mater. Sci. Eng. C Mater. Biol. Appl., 77, 955-962.

[8] Jelínek, M., Písařík, P., Kocourek, T., Zemek, J., Kotzianova, A., Jurek, K., Miksovsky, J., \& Luxbacher, T. (2014). Preliminary comparative study of laser-prepared DLC and Cr-doped DLC for bacteria adhesion. Applied Physics A, 116, 1437-1443.

[9] Jelínek, M., Kocourek, T., Zemek, J., Mikšovský, J., Kubinová, Š., Remsa, J., Kopeček, J., \& Jurek, K. (2015). Chromium-doped DLC for implants prepared by laser-magnetron deposition. Materials Science and Engineering: C, 46, 381-386.

[10] Jelinek, M., Zemek, J., Vandrovcova, M., Bacakova, L., Kocourek, T., Remsa, J., \& Pisarik, P. (2016). Bonding and bio-properties of hybrid/magnetron Cr-enriched DLC layers. Materials Science and Engineering: C, 58, 1217-1224.

[11] Filova, E., Vandrovcova, M., Jelinek, M., Zemek, J., Houdkova, J., Remsa, J., Stankova, L., \& Bacakova, L. (2015). Differentiation of saos-2 osteoblast-like cells on chromium-doped diamond-like carbon coatings. PLOS ONE.

[12] Písařík, P., Jelínek, M., Kocourek, T., Zezulová, M., Remsa, J., \& Jurek, K. (2014). Chromium-doped diamond-like diamond-like carbon films deposited by dual-pulsed laser deposition. Applied Physics A, 117(1), 83-88.

[13] Wang, Q., Zhou, F., Zhou, Z., Yang, Y., Yan, C., Wang, C., Zhang, W., Li, K-Y.L., Bello, I., \& Lee, S-T. (2012). Influence of Ti content on the structure and tribological properties of Ti-DLC coatings in water lubrication. Diam. Relat. Mater., 25, 163-75.

[14] Vandrovcová, M., Hanuš, J., Drabík, M., Kylián, O., Biederman, H., Lisá, V., \& Bačáková, L. (2012). Effect of different surface nanoroughness of titanium dioxide films on the growth of human osteoblast-like MG63 cells. J. Biomed. Mater. Res. A, 100, 1016-1032.

[15] Wachesk, C. C., Pires, C. A. F., Ramos, B. C., Trava-Airoldi, V. J., Lobo, A. O., Pacheco-Soares, C., Marciano, F. R., \& Da-Silva, N. S. (2013). Cell viability and adhesion on diamond-like carbon films containing titanium dioxide nanoparticles. Appl. Surf. Sci., 266, 176-181.

[16] Jelínek, M., Písařík, P., Kocourek, T., Zemek, J., \& Lukeš, J. (2013). Influence of ion bombardment on growth and properties of PLD created DLC films. Appl Phys A, 110, 943-947.

[17] Kocourek, T., Jelínek, M., Písař́ík, P., Remsa, J., Janovská, M., Landa, M., Zemek, J., \& Havránek, V. (2017). Diamond-like carbon layers modified by ion bombardment during growth and researched by resonant ultrasound spectroscopy. Applied Surface Science, 417, 213-217.

[18] Písařík, P., Mikšovský, J., Remsa, J., Zemek, J., Tolde, Z., \& Jelínek, M. (2017). Diamond like carbon prepared by pulsed laser deposition with ion bombardment - physical properties, presented at COLA 2017, Appl. Phys. A.

[19] Jelínek, M., Vaněk, P., Tolde, Z., Buixaderas, E., Kocourek, T., Studnička, V., Drahokoupil, J., Petzelt, J., Remsa, J., \& Tyunina, M., (2017). PLD prepared bioactive $\mathrm{BaTiO}_{3}$ films on TiNb implants. Materials Science and Engineering: C, 70(1), 334-339.

[20] Jelínek, M., Buixaderas, E., Drahokoupil, J., Kocourek, T., Remsa, J., Vaněk, P., Vandrovcová, M., Doubková, M., \& Bačáková, L. (2017). Laser-synthesized nanocrystalline, ferroelectric, bioactive $\mathrm{BaTiO}_{3} / \mathrm{Pt} / \mathrm{FS}$ for bone implants. Appl. Phys. A. 


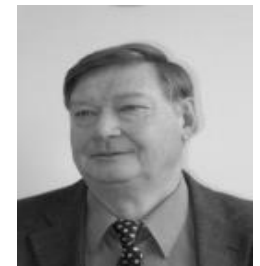

Miroslav Jelínek was born in 1946. He received Ph.D. degree from Czech Technical University in 1978, and degree of the doctor of science in 1999. From 2009 he is professor at CTU Prague. Since 1984 he has been with Institute of Physics ASCR, Prague. He is oriented on the development of new laser technologies, on the study of superlattices, graded layers, biocompatible layers, thermoelectric \{layers, and waveguides. He published more than 240 papers in refereed, impacted, international scientific journals. Hirsch index 25, 2430 citations.

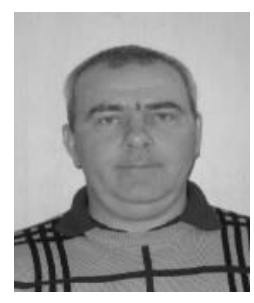

650 citations.

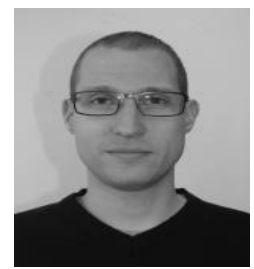

180 citations.

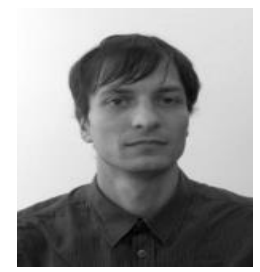

Petr Písařík was born in Benešov, the Czech Republic in 1985. He received his Ph.D. in biomedical and clinical technology from the Czech Technical University in Prague in 2016. Since 2010 (2012) he has been with Institute of Physics ASCR (Czech Technical University in Prague). His research interests include thin layers preparation and characterization (techniques: Pulsed Laser Deposition, Magnetron Sputtering, Radio-frequency discharge, MAPLE, Atomic Force Microscopy, Mechanical profilometry, Optical spectroscopies), laser and electronic devices maintenance. He published 14 papers in refereed, impacted, international scientific journals. Hirsch index 4, 62 citations.

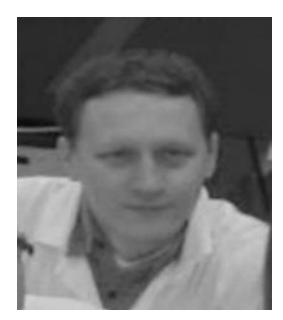

Jan Mikšovský was born in Vsetin, Czech Republic, 1980. He got the master degree received at Czech Technical University in the study field Instruments and methods for biomedicine; the Ph.D. degree received at Czech technical university in the study field biomedical and clinical technology, Prague, Czech republic in 2016.

He works as researcher at Institute of Physics, Czech Academy of Science, Prague and as teaching assistant at CTU, Faculty of Biomedical Engineering, Kladno. 